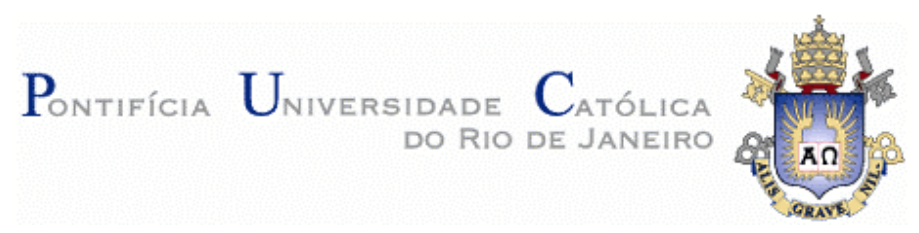

Edgar Adrian de Oliveira

\title{
Confiabilidade metrológica na determinação de espécies químicas em materiais siderúrgicos por espectrometria de fluorescência de raios- $X$.
}

Dissertação apresentada como requisito parcial para obtenção do título de Mestre pelo Programa de PósGraduação em Metrologia. Area de Concentração: Metrologia para Qualidade e Inovação.

Orientadores: Prof. Mauricio Nogueira Frota, PhD Prof. Ricardo Queiroz Aucelio, PhD Alessandra Licursi Maia Cerqueira da Cunha, Dr. 


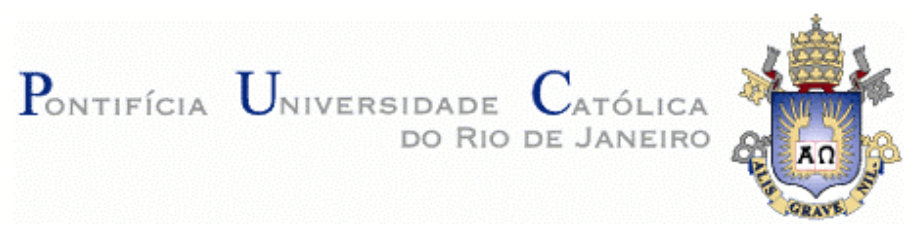

Edgar Adrian de Oliveira

\section{Confiabilidade metrológica na determinação de espécies} químicas em materiais siderúrgicos por espectrometria de

fluorescência de raios- $X$.

Dissertação apresentada como requisito parcial para obtenção do grau de Mestre pelo Programa de PósGraduação em Metrologia do Centro Técnico Científico da PUC-Rio. Aprovada pela Comissão Examinadora e homologada pela Coordenação Setorial de PósGraduação, formalizado pelas respectivas assinaturas:

Prof. Dr. Maurício Nogueira Frota (Orientador)

Programa de Pós-Graduação em Metrologia (PosMQI/PUC-Rio) Pontifícia Universidade Católica do Rio de Janeiro

Prof. Dr. Ricardo Queiroz Aucelio (Co-orientador)

Departamento de Química Pontifícia Universidade Católica do Rio de Janeiro

\section{Prof ${ }^{\text {. Dra }}$. Alessandra Licursi M. C. da Cunha (Co-orientadora) Departamento de Química Pontifícia Universidade Católica do Rio de Janeiro}

Prof ${ }^{\text {. Dra }}$. Elisabeth Costa Monteiro Programa de Pós-Graduação em Metrologia (PosMQI/PUC-Rio) Pontifícia Universidade Católica do Rio de Janeiro

Prof. Dr. Fabrício Casarejos Lopes Luiz Programa de Pós-Graduação em Metrologia (PosMQI/PUC-Rio) Pontifícia Universidade Católica do Rio de Janeiro

Coordenacão Setorial de Pós-Graduação:

Prof. José Eugenio Leal Coordenador Setorial de Pós-Graduação do Centro Técnico Científico -

(PUC-Rio) 
Todos os direitos reservados. É proibida a reprodução total ou parcial do trabalho sem autorização da universidade, do autor e do orientador.

\section{Edgar Adrian de Oliveira}

Graduado em 1999 como Engenheiro Químico pela (UFRRJ) *Universidade Federal Rural do Rio de Janeiro. Pós-graduado em 2001 na especialidade Química pela (UFLA) *Universidade Federal de Lavras. Engenheiro especialista do laboratório químico da aciaria da ArcelorMittal Tubarão, desde agosto de 2002.

Ficha Catalográfica

Oliveira, Edgar Adrian de

Confiabilidade metrológica na determinação de espécies químicas em materiais siderúrgicos por espectrometria de fluorescência de raios-X / Edgar Adrian de Oliveira ; orientadores: Mauricio Nogueira Frota, Ricardo Queiroz Aucelio, Alessandra Licursi Maia Cerqueira da Cunha. - 2011.

115 f. : il. (color.) ; $30 \mathrm{~cm}$

Dissertação (mestrado)-Pontifícia Universidade Católica do Rio de Janeiro, Programa de Pós-Graduação em Metrologia para a Qualidade e Inovação, 2011.

Inclui bibliografia

1. Metrologia - Teses. 2. Confiabilidade metrológica. 3. Fluorescência de raios-X. 4. Validação. 5. Materiais siderúrgicos. 6. Metrologia. 7. Controle de qualidade I. Frota, Mauricio Nogueira. II. Aucelio, Ricardo Queiroz. III. Cunha, Alessandra Licursi Maia Cerqueira da. IV. Pontifícia Universidade Católica do Rio de Janeiro. Programa de Pós-Graduação em Metrologia para a Qualidade e Inovação. V. Título.

CDD: 389.1 
A minha mulher Andréa pela total cumplicidade, atenção e amor.

Aos meus filhos Gabriel e Carolina por darem sentido a tudo.

Aos meus pais João e Angela e meus irmãos Ciro e Thiago por me presentearem com o conceito da família.

Aos meus sobrinhos João, Zé e Juju por me despertarem o olhar de pai. 


\section{Agradecimentos}

Ao meu orientador Prof. Maurício Frota pela compreensão, apoio, paciência e orientação.

Aos co-orientadores Prof. Ricardo Aucelio e Alessandra Licursi pela orientação.

A todos os meus professores que ao longo de minha vida acadêmica, com toda sua sabedoria, me fizeram caminhar, mesmo que por árduos caminhos, mas chegando sempre ao objetivo.

A ArcelorMittal Tubarão, principalmente aos gerentes do Departamento de Metalurgia e Planejamento de Produção, pelo apoio ao meu trabalho.

A todos os colegas de trabalho, aqui representados por Ulisses, Ronaldo, Sérgio e Hermógenes, pela paciência e suporte no desenvolvimento deste trabalho.

Ao querido Bazoni (in momoriam).

Ao amigo Alex, que me incentivou a iniciar e apoiou na conclusão deste projeto.

A minha equipe de trabalho Angela, Amanda, Carol, Laura, Marcos Barcelos, Marcos Roberto, Paim e Terezinha, pela leveza do trabalho diário e apoio durante minhas ausências.

Aos meus amigos Duda, Truly, Leo e Carla, por me receberem durante minhas viagens ao Rio.

Aos meus colegas de curso, principalmente Katia, Ana, Leo, Thiago, Wesley e Eliana, pelos momentos vividos durante esta jornada.

A professora Fátima Ludovico e as secretárias Márcia Ribeiro e Paula, pela apoio e paciência.

A todos os amigos e colegas que durante toda minha vida mostraram que a vida é um processo contínuo de opções em que acertar é muitas vezes difícil, mas tentar é essencial. 


\section{Resumo:}

Adrian de Oliveira, Edgar; Frota, Maurício Nogueira. Confiabilidade metrológica na determinação de espécies químicas em materiais siderúrgicos por espectrometria de fluorescência de raios-X. Rio de Janeiro, 2011. 115p. Dissertação de Mestrado - Programa de Pós-Graduação em Metrologia, Pontifícia Universidade Católica do Rio de Janeiro.

O objetivo da dissertação é avaliar a confiabilidade metrológica da técnica de fluorescência de raios-X utilizada em análises quantitativas de espécies químicas presentes em materiais siderúrgicos utilizados na produção do aço. $\mathrm{O}$ estudo da técnica e a avaliação da capacidade do equipamento analítico associado às etapas de recebimento, preparação e análise das amostras permitem definir os itens críticos de controle para monitoramento e garantia dos resultados emitidos. Como motivação, o interesse em garantir a aplicação de um método já conhecido às novas demandas de análises químicas, suprindo as necessidades dos clientes, otimizando-se, assim, os serviços prestados no laboratório responsável pelo processo de controle de qualidade da ArcelorMittal Tubarão. A metodologia de desenvolvimento do trabalho baseou-se nas boas práticas da metrologia, assegurando que medições sejam adequadamente realizadas, reduzindo o consumo e o desperdício de matéria-prima e gerando ganhos de produtividade. Em conformidade às recomendações do Guia EURACHEM, a validação da técnica levou em conta a confirmação do analito, seletividade/especificidade, limite de detecção, limite de quantificação, recuperação, intervalo de medição, linearidade, exatidão, veracidade, precisão (repetitividade, precisão intermediária) e robustez. Dentre os resultados, o trabalho valida o método para o trabalho de rotina, qualificando a fluorescência de raios-X como método de análise para os materiais analisados no laboratório. Como conclusão o estudo da metodologia de validação metrológica deixa uma fonte bibliográfica da técnica e do equipamento utilizados, de forma a difundir estes princípios. Outra contribuição do presente trabalho foi a possibilidade de expansão da referida técnica para outras análises químicas realizadas no laboratório da ArcelorMittal Tubarão.

\section{Palavras-chave:}

Confiabilidade Metrológica; Fluorescência de raios- $X$; Validação; Materiais Siderúrgicos; Metrologia; Controle de Qualidade 


\section{Abstract:}

Adrian de Oliveira, Edgar; Frota, Maurício Nogueira (Advisor). Metrological reliability of the analytical method by X-ray fluorescence for steel industry materials. Rio de Janeiro, 2011. 115p. MSc. Dissertation Programa de Pós-Graduação em Metrologia, Pontifícia Universidade Católica do Rio de Janeiro.

This master's degree dissertation purpose is evaluate, by means of metrological validation techniques, the reliability of the analytical method by Xray fluorescence, used in quantitative analysis of chemical species presents in various materials used in steel production process. The method study and the evaluation of the analytical equipment capacity associated with the correct understanding of the steps of receiving, preparation and analysis of samples will define the critical issues of control to guarantee the emitted results. As motivation, the interest for ensuring the implementation of a method already known in the new demands of chemical analysis, supplying the needs of customers, optimizing, thus, the services provided in the laboratory responsible for quality control of ArcelorMittal Tubarão. The methodological study development was based on good practice of metrology in order to ensure adequate measurements made during a production process, reducing consumption and waste of raw materials and generating productivity gains. In accordance with the EURACHEM Guide, the method validation took into account the Confirmation of identity, Selectivity / Specificity, Limit of detection, Limit of quantification, Recovery, Working and linear ranges, Accuracy, Trueness, Precision (repeatability, intermediate precision) and Robustness. Among the results, the study validates the method for routine work, qualifying X-ray fluorescence as a method of analysis for materials tested in the laboratory. As conclusion the study of the metrological validation methodology leaves a bibliographic source of the method and equipment used, in order to spread these principles. Another contribution of this work was the possibility of expanding this technique to other analysis realized in the laboratory of ArcelorMittal Tubarão.

\section{Keywords:}

Metrological Reliability; X-Ray Fluorescence; Validation; Steel Industry Products; Metrology; Quality Control 


\section{Sumário}

1 Considerações iniciais 13

1.1. Objetivo 15

1.2. Motivação 15

$\begin{array}{ll}\text { 1.3. Estrutura da dissertação } & 15\end{array}$

20 papel da metrologia 17

2.1. Conceitos básicos 17

2.2. Origem da metrologia em química no Brasil 18

3 A ArcelorMittal Tubarão 20

3.1. Breve histórico 20

3.2. O fluxo de produção da ArcelorMittal Tubarão 22

$\begin{array}{ll}\text { 3.3. Controle de qualidade } & 25\end{array}$

4 Geração de amostra objeto de uma análise química e seu processo $\begin{array}{ll}\text { de preparação } & 30\end{array}$

4.1. Estabelecendo um método de amostragem 30

4.2. Princípios de preparação de uma amostra para análise por fluorescência de raios-X 34

4.3. Método de preparação de amostras 35

4.4. Equipamentos utilizados na preparação de amostras 39

5 Fundamentos teóricos da técnica de análise espectrométrica por fluorescência de raios-x 43

5.1. Introdução 43

5.2. Descoberta dos raios- $X \quad 43$

5.3. O princípio da espectrometria de fluorescência de raios- $X \quad 44$

5.4. Radiações características 51

5.5. Detecção 54

5.6. Intensidades de raios-X 55 
60 espectrômetro de fluorescência de raios-x Thermo ARL 990058

6.1. O tubo de raios-X 58

6.2. Dispersão 62

6.3. Componentes internos 64

6.4. Detecção 73

6.5. Interpretação das informações do detector 81

7 Avaliação metrológica e potencial de aplicação da fluorescência de raios-x em amostras de um processo siderúrgico 82

7.1. Confiabilidade metrológica 82

7.2. Controle metrológico $\quad 84$

7.3. Avaliação do método 85

7.4. Potencial de aplicação da técnica por fluorescência de raios-X 107

8 Conclusão 110

9 Bibliografia 112

10 Anexos 115 


\section{Lista de figuras}

Figura 1. Vista aérea e localização da ArcelorMittal Tubarão 22

Figura 2. Diagrama do fluxo de produção da ArcelorMittal Tubarão 25

Figura 3. Organograma do laboratório químico da aciaria 27

Figura 4. Interface entre o laboratório e as áreas clientes 27

Figura 5. Tipos de constituintes vs. concentração do analito 31

Figura 6. Classificação dos analitos vs. dimensão da amostra. 32

Figura 7. Representação do raio X 44

Figura 8. Geração de um fóton de raios-X 46

Figura 9. Efeito Auger 47

$\begin{array}{ll}\text { Figura 10. Rendimento da fluorescência } & 48\end{array}$

Figura 11. Espalhamento compton $\quad 49$

Figura 12. Relação entre o background e o espalhamento compton $\quad 50$

Figura 13. Transições eletrônicas em um átomo de cálcio 52

Figura 14. Espectro típico de um WDS 53

$\begin{array}{ll}\text { Figura 15. Espectrômetro WDX } & 58\end{array}$

Figura 16. Espectro de um tubo de Rh $\quad 59$

$\begin{array}{ll}\text { Figura 17. Intensidade X comprimento de onda } & 60\end{array}$

Figura 18. Geometria de um XRF seqüencial 63

$\begin{array}{ll}\text { Figura 19. Geometria de um XRF simultâneo } & 64\end{array}$

Figura 20. Sistema de um Goniômetro $\quad 65$

Figura 21. Relação entre colimadores, resolução e sensibilidade 66

$\begin{array}{ll}\text { Figura 22. Planos cristalográficos em um cristal cúbico } & 67\end{array}$

$\begin{array}{ll}\text { Figura 23. Difração de raios-X } & 67\end{array}$

Figura 24. Relação entre difração e dispersão $\quad 71$

Figura 25. Detectores selados $\quad 74$

$\begin{array}{ll}\text { Figura 26. Região de avalanche } & 76\end{array}$

$\begin{array}{ll}\text { Figura 27. Contador de cintilação } & 79\end{array}$

Figura 28. Pulsos de diferentes ordens $\quad 80$

$\begin{array}{ll}\text { Figura 29. Ajuste da janela para seleção de um pulso } & 81\end{array}$

Figura 30. Processo de desenvolvimento de um método. $\quad 88$

Figura 31. Parâmetros de controle do equipamento Thermo ARL 9900 


\section{Lista de tabelas}

Tabela 1. Configuração das áreas analíticas do laboratório 28

Tabela 2. Métodos típicos de preparação de amostras 36

Tabela 3. Linhas espectrais típicas 56

Tabela 4. Lista de procedimentos do LQA 85

Tabela 5. Parâmetros analíticos e seus requisitos de desempenho. 89

Tabela 6. Avaliação da confirmação do analito (valores em \% p/p) 90

Tabela 7. Avaliação da seletividade (valores em \% p/p) 91

Tabela 8. Avaliação do limite de quantificação (valores em \% p/p) 93

Tabela 9. Avaliação da recuperação (valores em \% p/p) 94

Tabela 10. Avaliação do Intervalo de Medição (valores em \% p/p) 95

Tabela 11. Determinação da linearidade através da análise do resíduo 97

Tabela 12. Avaliação da Veracidade (valores em \% p/p) 99

Tabela 13. Avaliação da Repetitividade 102

Tabela 14. Condições de análise do estudo de precisão intermediária. 103

Tabela 15. Avaliação da Precisão Intermediária 104

Tabela 16 Materiais analisados por fluorescência de raios-X na TMTL 106

$\begin{array}{ll}\text { Tabela 17. Potenciais de migração de análise para raios-X } & 108\end{array}$ 


\section{Lista de Abreviaturas e Símbolos}

\begin{tabular}{|c|c|}
\hline $\mathbf{A B M}$ & Associação Brasileira de Metalurgia, Materiais e Mineração \\
\hline AMT & ArcelorMittal Tubarão \\
\hline BIPM & Bureau Internacional de Pesos e Medidas \\
\hline CBM & Comitê Brasileiro de Metrologia \\
\hline CCQM & Conselho Consultivo para Quantidade de Matéria \\
\hline CONMETRO & $\begin{array}{l}\text { Conselho Nacional de Metrologia, Normalização e Qualidade } \\
\text { Industrial }\end{array}$ \\
\hline CST & Companhia Siderúrgica de Tubarão \\
\hline ECRM & Euronorm Certified Reference Materials \\
\hline INMETRO & $\begin{array}{l}\text { Instituto Nacional de Metrologia, Normalização e Qualidade } \\
\text { Industrial }\end{array}$ \\
\hline IPT & Instituto de Pesquisas Tecnológicas \\
\hline ISO & International Organization for Standardization \\
\hline LQA & Laboratório Químico da Aciaria \\
\hline LTQ & Laminador de Tiras a Quente \\
\hline MRC & Material de Referência Certificado \\
\hline OHSAS & Occupational Health \& Safety Advisory Services \\
\hline PBQM & Programa Brasileiro de Metrologia em Química \\
\hline PNCO & Programa Nacional de Certificação de Operadores \\
\hline SCMQ & Sub Comitê de Metrologia em Química \\
\hline SEBRAE & Serviço Brasileiro de Apoio às Micro e Pequenas Empresas \\
\hline VIM & $\begin{array}{l}\text { Vocabulário Internacional de Termos Fundamentais e Gerais de } \\
\text { Metrologia }\end{array}$ \\
\hline XRD & X Ray Diffraction \\
\hline XRF & X Ray Fluorescence \\
\hline
\end{tabular}

